\title{
Mapping Choroidal and Retinal Thickness Variation in Type 2 Diabetes using Three-Dimensional 1060-nm Optical Coherence Tomography
}

\author{
Marieh Esmaeelpour, ${ }^{1,2,3}$ Boris Považay, ${ }^{3}$ Boris Hermann, ${ }^{3}$ Bernd Hofer, ${ }^{3}$ Vedran Kajic, ${ }^{1}$ \\ Sarab L. Hale, ${ }^{4}$ Rachel V. North, ${ }^{1}$ Wolfgang Drexler, ${ }^{3}$ Nik J. L. Sheen ${ }^{1}$
}

Purpose. To map choroidal (ChT) and retinal thickness (RT) in healthy subjects and patients with diabetes with and without maculopathy using three dimensional 1060-nm optical coherence tomography (3D-1060nm-OCT).

Methods. Sixty-three eyes from 42 diabetic subjects (41-82 years of age; 11 females) grouped according to a custom scheme using Early Treatment Diabetic Retinopathy Study definitions for pathology within 1 disc-diameter of fovea (without pathology [NDR], microaneurysms [M1], exudates [M2], clinically significant macular edema [CSME]) and 16 eyes from 16 healthy age matched subjects (38-79 years of age; 11 females) were imaged by 3D-1060nm-OCT performed over a $36^{\circ} \times 36^{\circ}$ field of view. Axial length, $45^{\circ}$ fundus photographs, body mass index, plasma glucose, and blood pressure measurements were recorded. The ChT at the subfoveal location and ChT maps between RPE and the choroidal-scleral interface were generated and statistically analyzed.

REsults. RT maps show thinning in the NDR group but an increase in thickness with increasing maculopathy in the temporal and central regions (unpaired $t$-test; $P<0.05$ ). ChT mapping of all diabetic patients revealed central and inferior thinning compared to healthy eyes (unpaired $t$-test; $P<0.001$ ). Subfoveal ChT (mean \pm SD) for healthy eyes was $327 \pm 74$ $\mu \mathrm{m}$, which was significantly thicker than all diabetic groups $(214 \pm 55 \mu \mathrm{m}$ for NDR, $208 \pm 49 \mu \mathrm{m}$ for M1, $205 \pm 54 \mu \mathrm{m}$ for M2, and $211 \pm 76 \mu \mathrm{m}$ for CSME (ANOVA $P<0.001$; Tukey $P<0.001)$.

Conclusions. 3D-1060nm-OCT has shown that the central choroid is thinner in all type 2 diabetic eyes regardless of disease stage. The choroidal thinning may exceed the magni-

From the ${ }^{1}$ School of Optometry and Vision Sciences, Cardiff University, United Kingdom; ${ }^{2}$ Ludwig Boltzmann Institute for Retinology and Biomicroscopic Lasersurgery, Department of Ophthalmology, Rudolf Foundation Clinic, Vienna, Austria; ${ }^{3}$ Center for Medical Physics and Biomedical Engineering, Medical University Vienna, Austria; and the ${ }^{4}$ Department of Ophthalmology, University Hospital of Wales, Cardiff, United Kingdom.

Supported in part by the Biotechnology and Biological Sciences Research Council, Cardiff University; FP6-IST-NMP-2 STREPT (017128, NanoUB); DTI Grant (OMICRON); AMR Grant (AP1110); European Union project FUN OCT (FP7 HEALTH, contract no. 201880) and Carl Zeiss Meditec Inc.; and Diabetes UK Grant BDA:RD07/003472.

Submitted for publication November 11, 2010; revised February 25, 2011; accepted March 15, 2011

Disclosure: M. Esmaeelpour, None; B. Považay, None; B. Hermann, None; B. Hofer, None; V. Kajic, None; S.L. Hale, None; R.V. North, None; w. Drexler, Carl Zeiss Meditec (C); N.J.L. Sheen, None

Corresponding author: Marieh Esmaeelpour, General Hospital Vienna, 4L Waehringer Guertel 18-20, Vienna 1090, Austria;

marieh.esmaeelpour@meduniwien.ac.at. tude of possible choriocapillaris atrophy. In contrast to the conventional assessment of pathologic thickness change in several locations, thickness maps allow investigation of the choroid over the extent of affected areas. (Invest Ophthalmol Vis Sci. 2011;52:5311-5316) DOI:10.1167/iovs.10-6875

$\mathrm{T}$ he principal changes in diabetic eye disease are the breakdown of the blood-retinal barrier, retinal vasculature integrity $^{1}$ and hemodynamic abnormalities. ${ }^{2}$ Development of ischemia in the macula caused by capillary nonperfusion is correlated with vision loss. ${ }^{3}$ However, because of the high metabolic demand of the photoreceptors, the supply of glucose and oxygen to the macular region is largely provided by the choroid. ${ }^{4}$ Despite the choroid being an integral part of the nutrient and oxygen exchange with the retina, structural changes in the choroid and their potential effects on retina tissue have not previously been investigated in the living eye. Histologic evidence has shown that diabetic retinopathy causes atrophy of the choriocapillaris endothelium, ${ }^{5}$ but examination of these structural changes in vivo have been hampered by the limitations of commercial optical coherence tomography (OCT) instruments in imaging the choroid-sclera boundary in patients with thick choroids or obtaining an adequate field of view because of their operation in the 800-nm wavelength band. ${ }^{6-8}$ Recently, OCT at $1060 \mathrm{~nm}$ has shown the ability to visualize the choroid because of less scattering caused by the RPE, ${ }^{9}$ thereby allowing human choroid thickness (ChT) to be measured in healthy subjects. ${ }^{10,11}$ These studies found that ChT varies with age ${ }^{11}$ and axial length, ${ }^{10}$ and suggests that it could be a reliable biomarker for retinal eye disease.

The aims of this study are to image the choroid in healthy normal subjects compared to various stages of diabetic eye disease, mapping its thickness and documenting associated retinal changes by using retinal thickness (RT) maps.

\section{Methods}

\section{Subjects}

Forty-two subjects with type 2 diabetes mellitus and 16 healthy subjects were recruited. Ethical approval was obtained from the School of Optometry and Vision Sciences, Cardiff University, Ethics Committee, and the Central and Local Office for Research Ethics Committees. The studies followed the Declaration of Helsinki, and informed consent was obtained from all subjects before participation. Exclusion criteria were systemic diseases other than diabetes, history of retinal surgery, retinal pathology such as glaucoma and AMD, or physiological variation from normal. Inclusion criteria were a previous diagnosis of diabetes type 2. Demographic data of the subject cohort can be found in Table 1. Retinal status of diabetic eyes was evaluated by an experienced ophthalmologist with slit lamp biomicroscopy with a Volk lens. All lesions were recorded and eyes were graded according to Early Treatment 
TABLE 1. Demographic and Statistical Data of the Cohort

\begin{tabular}{|c|c|c|c|c|c|c|c|c|}
\hline & $\begin{array}{l}\text { Age } \\
(y)\end{array}$ & $\underset{(\mathbf{m m})}{\mathbf{A L}}$ & $\begin{array}{c}\text { Casual Plasma } \\
\text { Glucose }(\mathrm{mmol} / \mathrm{L})\end{array}$ & $\begin{array}{c}\mathrm{BMI}^{*} \\
\left(\mathrm{~kg} / \mathrm{m}^{2}\right)\end{array}$ & LogMAR & $\begin{array}{c}\text { SBP } \\
(\mathrm{mm} \mathrm{Hg})\end{array}$ & $\begin{array}{c}\text { DBP } \\
(\mathrm{mm} \mathrm{Hg})\end{array}$ & $\begin{array}{l}\text { Duration } \\
\quad(y)\end{array}$ \\
\hline \multicolumn{9}{|c|}{ NDR $(n=15 ;$ males $=10 ;$ BP medication $=11)$} \\
\hline Mean $\pm S D$ & $69 \pm 10$ & $23.96 \pm 0.79$ & $9 \pm 4.1$ & $28.5 \pm 5$ & $0.09 \pm 0.12$ & $144 \pm 15$ & $87 \pm 11$ & $11 \pm 9$ \\
\hline Range & $45-81$ & $23.09-25.59$ & $3.8-17.3$ & $21.4-38.9$ & $-0.1-0.7$ & $120-174$ & $69-101$ & $5-40$ \\
\hline \multicolumn{9}{|c|}{ M1 $(n=19 ;$ males $=15 ;$ BP medication $=13)$} \\
\hline Mean $\pm S D$ & $63 \pm 14$ & $23.76 \pm 0.63$ & $10 \pm 6$ & $27.6 \pm 5.7$ & $0.14 \pm 0.16$ & $137 \pm 24$ & $82 \pm 15$ & $16 \pm 6$ \\
\hline Range & $42-82$ & $22.05-24.81$ & $3.8-22.1$ & $15.8-35.6$ & $-0.1-0.44$ & $99-182$ & 58-109 & $6-25$ \\
\hline \multicolumn{9}{|c|}{ M2 $(n=17 ;$ males $=13 ;$ BP medication $=15)$} \\
\hline Mean \pm SD & $58 \pm 13$ & $23.94 \pm 0.8$ & $9.9 \pm 6.1$ & $30.3 \pm 6.5$ & $0.12 \pm 0.19$ & $137 \pm 20$ & $78 \pm 14$ & $17 \pm 11$ \\
\hline Range & $40-79$ & $22.45-24.95$ & $4.2-27.8$ & $21-40.1$ & $-0.1-0.54$ & $99-171$ & 58-106 & $6-40$ \\
\hline \multicolumn{9}{|c|}{$\operatorname{CSME}(n=12 ;$ males $=10 ;$ BP medication $=9)$} \\
\hline Mean \pm SD & $68 \pm 12$ & $23.41 \pm 0.8$ & $10.5 \pm 4.7$ & $29.6 \pm 2.8$ & $0.18 \pm 0.16$ & $137 \pm 24$ & $80 \pm 14$ & $15 \pm 7$ \\
\hline Range & $41-80$ & $22.4-24.51$ & $3.7-19.4$ & $25.7-34.8$ & $-0.06-0.5$ & $82-171$ & $64-106$ & $1-25$ \\
\hline \multicolumn{9}{|c|}{ Healthy $(n=16 ;$ males $=6 ;$ BP medication $=5)$} \\
\hline Mean $\pm S D$ & $61.31 \pm 12$ & $23.39 \pm 0.8$ & & $24.3 \pm 3.4$ & $0.06 \pm 0.1$ & $131 \pm 23$ & $79 \pm 9$ & \\
\hline Range & $38-79$ & $22.06-25.01$ & & $16.3-33.1$ & $-0.16-0.24$ & 98-186 & $66-92$ & \\
\hline
\end{tabular}

AL, axial eye length; BMI, body mass index; BP, blood pressure; CSME, clinically significant macula edema; DBP, diastolic blood pressure; M1, microaneurysms within 1 disc-diameter of the fovea; M2, exudates within 1 disc-diameter; LogMAR, logarithm of the Minimum Angle of Resolution; NDR, without pathology; SBP, systolic blood pressure; SD, standard deviation.

${ }^{*}$ Univariate ANOVA $(P=0.02)$. Tukey test $(P=0.01)$ for healthy and M2.

Diabetic Retinopathy Study (ETDRS). ${ }^{12}$ For the study, maculopathy was prioritized over retinopathy when grouping the eyes and a greater differentiation between macula grades was made. The imaged field of view was an area centered on the fovea for comparison purposes. A custom scheme of classifications for grouping the diabetic eyes in this study was defined, which served the purpose of investigating the effect of DR and DME abnormalities on the choroid. The grading used basic ETDRS definitions of DR and DME abnormalities, in particular the definition for "CSME." The diabetic eyes were grouped for pathology within 1 disc-diameter of fovea (without pathology [NDR], microaneurysms [M1], exudates [M2], and clinically significant macular edema [CSME]). This resulted in a total of 63 eyes from subjects with diabetes. The distribution of diabetic retinopathy for these eyes is shown in Table 2.

Healthy control subjects were included only if they had fewer than three of the following risk factors for diabetes: family history of diabetes, age $>50$ years, ethnic background, diagnosed high blood pressure, heart attack, or stroke. Only nonsmokers were included. Only one eye was included per healthy subject. Healthy subjects and eyes were chosen to match age and axial lengths according to the mean and $\mathrm{SD}$ of the diabetic groups (ANOVA; $P>0.05$ ).

\section{Study Protocol}

Monocular visual acuity was determined with ETDRS Original Series Charts (Precision Vision, La Salle, IL). Retinal status was documented with a fundus photograph with a $45^{\circ}$ field centered on the macula (CR-DGi nonmydriatic retinal camera; Canon, Lake Success, NY) after pupil dilation. Five axial eye length (AL) measurements were averaged from each eye using optical biometry (IOL Master Zeiss; Jena, Germany). All subjects had their blood pressure (BP) measured after at least 20 minutes resting in a sitting position. All diabetic subjects had their casual blood glucose tested. Fasting blood glucose was not

TABLE 2. Number of Eyes with Diabetic Retinopathy for Each Maculopathy Grade

\begin{tabular}{lcccc}
\hline Maculopathy Grade & $\begin{array}{c}\text { NDR } \\
(\boldsymbol{n}=\mathbf{1 5})\end{array}$ & $\begin{array}{c}\text { M1 } \\
(\boldsymbol{n}=\mathbf{1 9})\end{array}$ & $\begin{array}{c}\text { M2 } \\
(\boldsymbol{n}=\mathbf{1 7})\end{array}$ & $\begin{array}{c}\text { CSME } \\
(\boldsymbol{n}=\mathbf{1 2})\end{array}$ \\
\hline No DR & 15 & - & - & - \\
Background DR & - & 14 & 12 & 9 \\
Pre-proliferative DR & - & 5 & 5 & 2 \\
Proliferative DR & - & - & - & 1 \\
\hline
\end{tabular}

CSME, clinically significant macula edema; DR, diabetic retinopathy; M1; microaneurysms; M2, exudates; NDR, without pathology. obtained, because this would have altered homeostatic mechanisms and not allowed a representative comparison to be made between normal and diabetic eyes. Information about diabetes duration, weight, and height were recorded.

\section{OCT Imaging and Thickness Maps}

High speed three-dimensional OCT imaging at $1060 \mathrm{~nm}$ (3D-1060nmOCT) was performed with $<2.5 \mathrm{~mW}$ at the cornea, well below the maximum power limit for 10 seconds of exposure. ${ }^{13,14}$ Three-dimensional OCT volumes were acquired at $1060 \mathrm{~nm}$ with 15 to $20 \mu \mathrm{m}$ transverse resolution, approximately $7 \mu \mathrm{m}$ axial resolution and 512 voxels per depth-scan (A-scan). Scans across a $36^{\circ} \times 36^{\circ}$ field at 47,000 A-scans per second were centered on the fovea and resulted in up to 70 frames per second. For image processing and manual measurement of pixel distances in choroid and retina, Image software was used (developed by Wayne Rasband, National Institutes of Health, Bethesda, MD; available at http://rsb.info.nih.gov/ij/index.html). ${ }^{5}$

The measurement of $\mathrm{ChT}$ and the generation of thickness maps is described elsewhere. ${ }^{10}$ Briefly, axial RT was defined as the distance between internal limiting membrane and the center of the peaks originating from the RPE/Bruch's membrane/ choriocapillaris (RBC) complex and axial $\mathrm{ChT}$ as the distance between RBC complex and the

TABLE 3. Retinal Foveal and Choroidal Subfoveal Thickness Measurements from Healthy Eyes and Eyes of Subjects with Diabetes

\begin{tabular}{|c|c|c|c|c|c|}
\hline & \multicolumn{4}{|c|}{ Range of Diabetic Maculopathies } & \multirow[b]{2}{*}{$\begin{array}{c}\text { Healthy } \\
\text { Eyes }\end{array}$} \\
\hline & NDR & M1 & M2 & CSME & \\
\hline \multicolumn{6}{|c|}{ Retina, $\mu \mathrm{m}$} \\
\hline Mean $\pm S D$ & $209 \pm 15$ & $221 \pm 32$ & $223 \pm 42$ & $282 \pm 72^{*}$ & $212 \pm 28$ \\
\hline Range & $189-238$ & 179-292 & $152-304$ & $172-374$ & $171-277$ \\
\hline \multicolumn{6}{|l|}{ Choroid, $\mu \mathrm{m}$} \\
\hline Mean $\pm S D$ & $214 \pm 55$ & $208 \pm 49$ & $205 \pm 54$ & $211 \pm 76$ & $327 \pm 74 \dagger$ \\
\hline Range & $114-365$ & $122-313$ & $122-321$ & $117-365$ & $207-510$ \\
\hline
\end{tabular}

CSME, clinically significant macula edema; M1; microaneurysms; M2, exudates; NDR, without pathology; SD, standard deviation.

* Significantly different from the healthy retinal thickness (ANOVA, $P<0.001$; Tukey test, $P<0.01$ ).

† Significantly different from the choroidal thickness in the diabetic groups (ANOVA, $P<0.001$; Tukey test, $P<0.001$ ). 
choroidal-scleral interface. For the investigation of the thickness variation throughout the entire field of view, thickness maps were generated based on manual segmentation. The resulting pixel distance was converted into optical distance using the depth sampling calibration for the 3D-1060nm-OCT system and further to the anatomic distance. This resulted in thickness maps for individual eyes. Individual ChT maps were viewed at the location of recorded microaneurysms, exudates, and focal edema for obvious changes in thickness.

For the statistical analysis of mean and variation, all the individual ChT and RT maps were aligned to each other in respect to the macula and optic nerve position with commercially available software (MATLAB; The MathWorks, Inc., Natick, MA). ChT maps of diabetic eyes were grouped by the category of maculopathy. Unreliable portions of the images with fewer than five measurements at one location were excluded. Mean and SD was obtained to create a compound map of average thickness for these five groups of eyes with color-coded thickness. The coefficient of variation was used to map the variation within each group. Difference maps were generated to investigate the change in ChT and RT by subtracting each diabetic category from the healthy eyes group. An additional statistical analysis of the difference between the healthy and each diabetic category was generated by conducting t-tests for each individual axial thickness over the field of view. Contour lines for $P>0.05$ and 0.001 were drawn on the difference maps to show areas of statistically significant difference. For statistical assessment of individual variation between eyes, central ChT measurement was located beneath the foveola.

\section{Results}

There was no difference between the subject groups in BP and logMAR vision and no difference between the diabetic subject groups for diabetes duration, $\log$ MAR, casual blood glucose and body mass index (BMI; Table 1; ANOVA, $P>0.05$ ). Only M2s had a significantly higher BMI than healthy subjects (post hoc Tukey test, $P<0.01$ )

All tomograms were of sufficient quality to allow ChT measurement. Mean subfoveal ChT was thickest for the healthy eyes (Table 3) and significantly different from all other groups (ANOVA, $P<0.001$; Tukey test, $P<0.001$ ). There was no
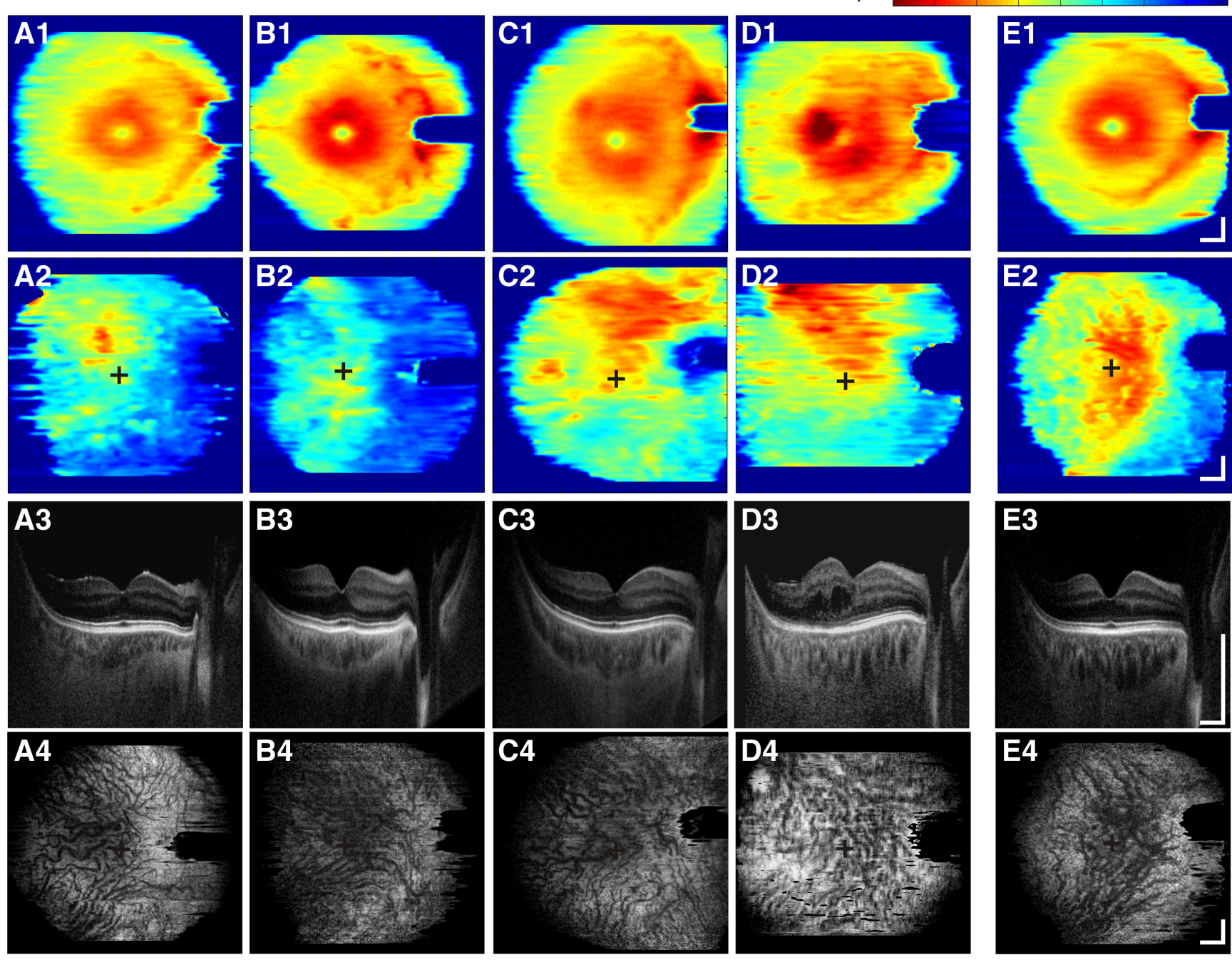

Figure 1. Examples in diabetic (A-D) and healthy (E) subjects of retinal thickness maps (1), choroidal thickness maps (2), tomograms through the center of the fovea and optic nerve (3), and enface tomograms through the Haller's and Sattler's layer of the choroid (4). Subjects with diabetes had different stages of maculopathy: NDR (A), M1 (B), M2 (C), and CSME (D). All subjects had short axial eye length (A, $23.09 \mathrm{~mm}, 71$ years of age; $\mathrm{B}, 23.34 \mathrm{~mm}, 76$ years of age; $\mathrm{C}, 22.78 \mathrm{~mm}, 47$ years of age; $\mathrm{D}, 22.42 \mathrm{~mm}, 55$ years of age; $\mathrm{E}, 22.06 \mathrm{~mm}, 66$ years of age). White lines in the horizontal represent $1 \mathrm{~mm}$ scale bars (for row $3,0.5 \mathrm{~mm}$ vertically). 

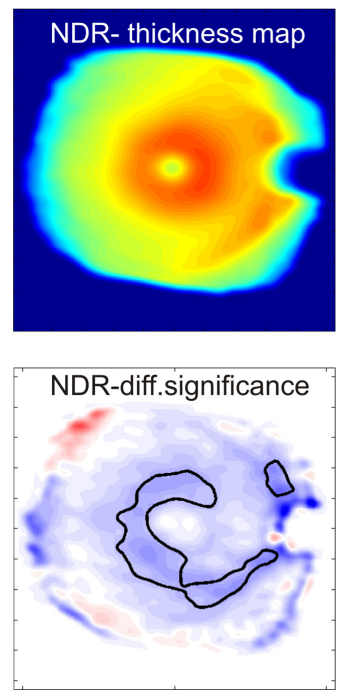
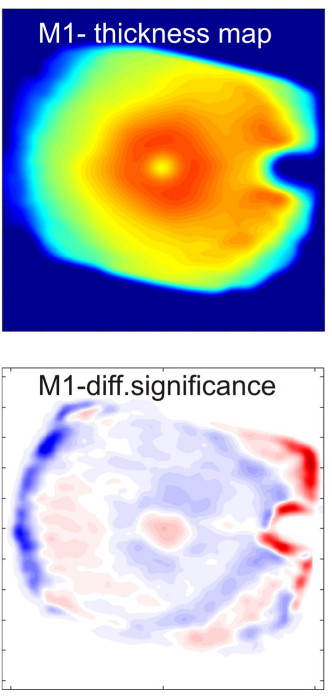
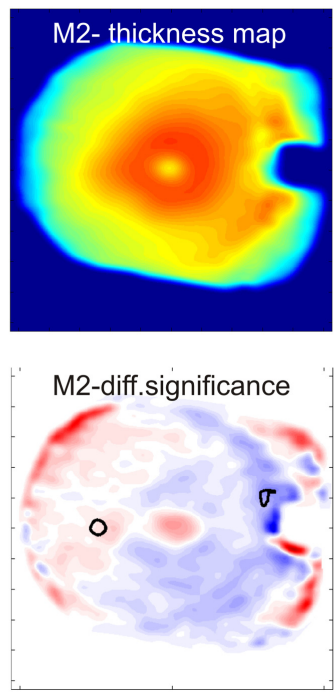
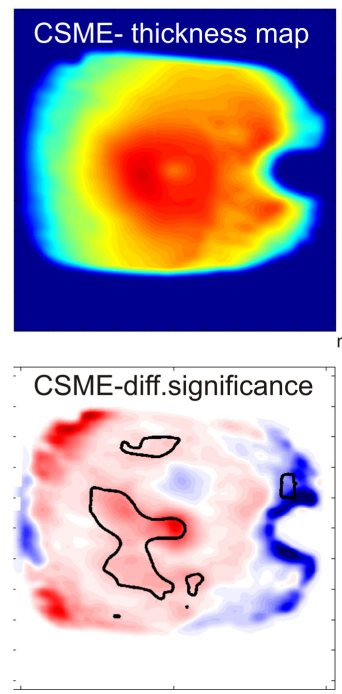
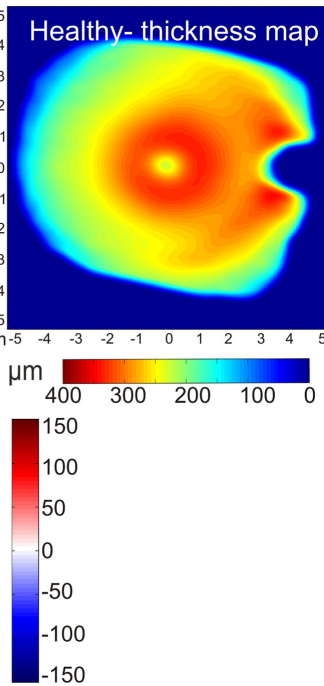

FigURE 2. Retinal thickness maps averaged for each study group (upper row) and a comparison of thickness change mapped with significant difference contour lines (lower row). Dotted lines represent statistical significance $(P=0.05)$. Solid lines represent statistical significance $(P=$ $0.05)$.

difference between the maculopathy groups. There was no apparent ChT decrease directly below the lesions. For a comparison between healthy and diabetic eyes, thickness maps proved to be easier to interpret than single tomograms (Fig. 1). The images from the 3D-1060nm-OCT were useful to investigate overall vessel distribution in enface view through the Haller's and Sattler's layer (Fig. 1, A4-E4).

Figure 2 shows averaged RT maps and difference maps with regions of significant difference between each pathology group and the healthy eyes. The comparison of averaged RT maps by groups (Fig. 2) confirms that the CSME group had the thickest macula; significantly centrally, temporally and inferiorly. The M2 retinal map had a small region of significant thickening temporally. The M1 group showed that the retina was thicker temporally but not significant in comparison with the healthy group, with only small areas of significant thinning above and below the optic nerve. The NDR group showed large regions of significant thinning just outside the macular region and in the region of the arcades.
Figure 3 shows ChT maps and difference maps with regions of significant difference between each pathology group and the healthy eyes. The NDR group shows an overall decrease $(P<0.001)$ in ChT with more significant decrease $(P<0.001)$ centrally, inferiorly, and temporally. The M1 group shows an extensive significant decrease $(P<0.001)$ in all regions of the choroid. The M2 group also shows an overall ChT decrease $(P<0.05)$, with a significant decrease $(P<0.001)$ inferiorly. Finally, in the CSME groups, ChT is decreased overall $(P<$ $0.05)$ in the far superior temporal periphery and inferiorly and superiorly nasally.

Contour lines delineating coefficient of variation changes show that large regions within the averaged thickness maps have low coefficient of variation $<30 \%$ (Fig. 4).

\section{Discussion}

The present study is a proof of concept that shows the feasibility of investigating the choroid in diabetic subjects. Healthy
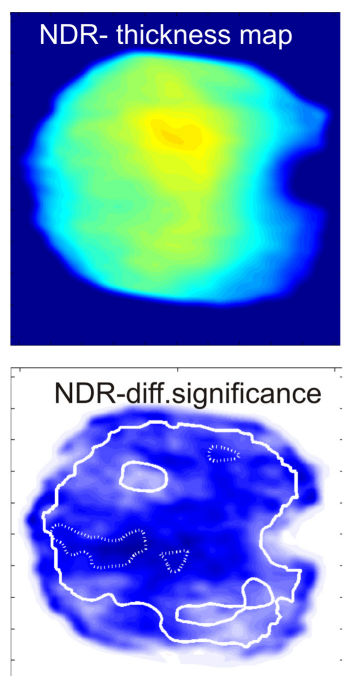
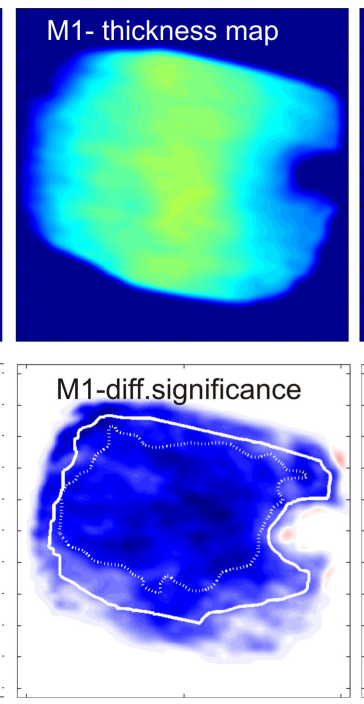
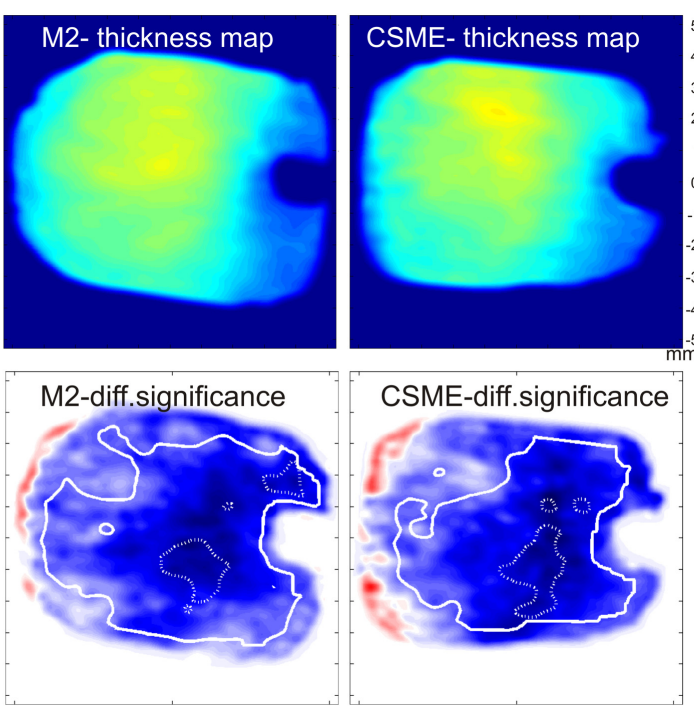
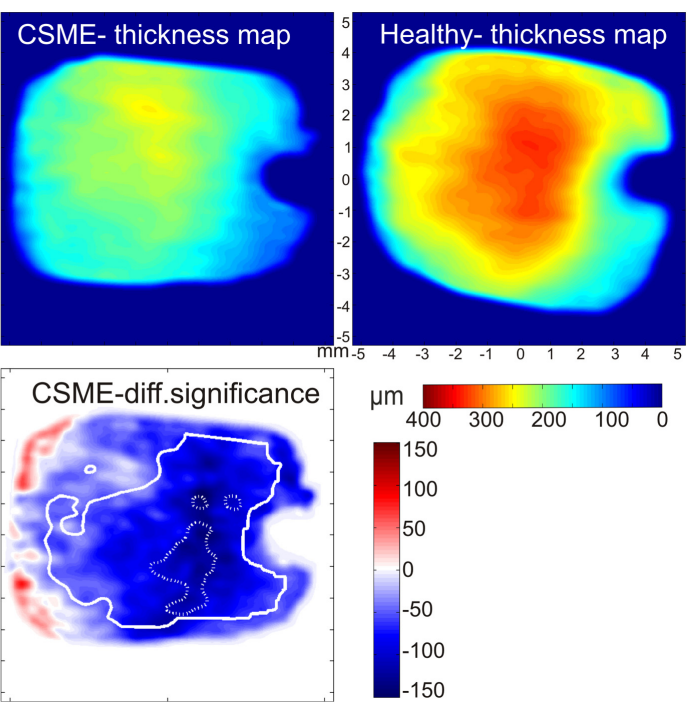

FIGURE 3. Choroidal thickness maps averaged for each study group (upper row) and a comparison of thickness change mapped with significant difference contour lines (lower row). Solid contour lines represent statistical significance $(P=0.05)$; dotted lines represent $P=0.001$. 

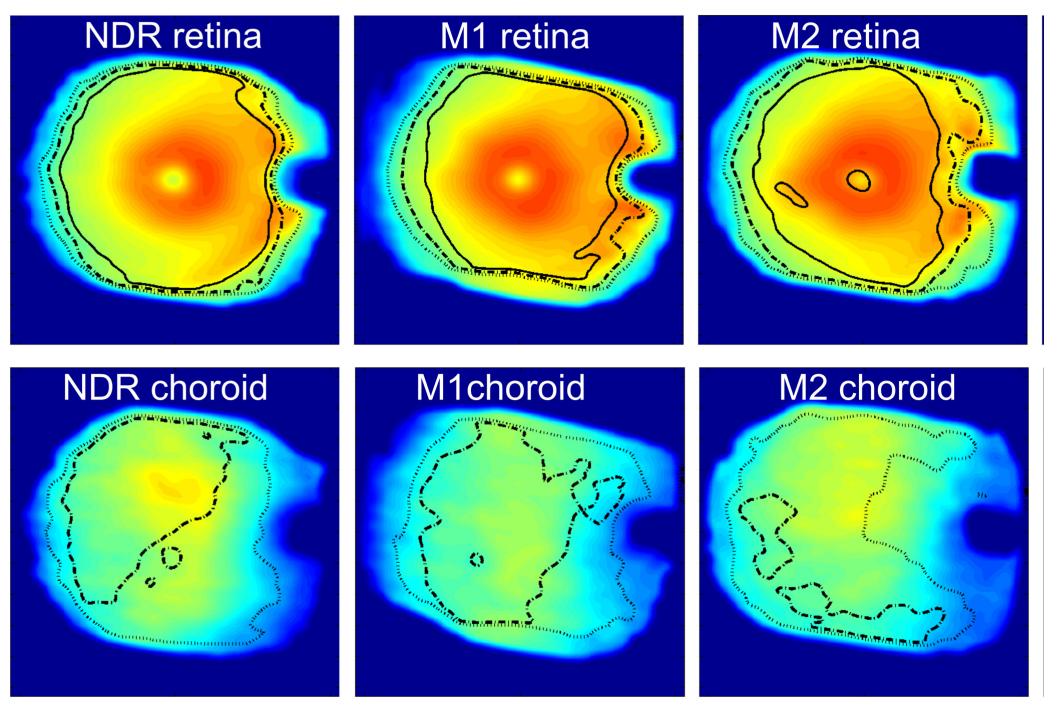

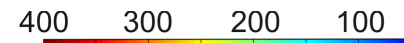

$\mu \mathrm{m}$
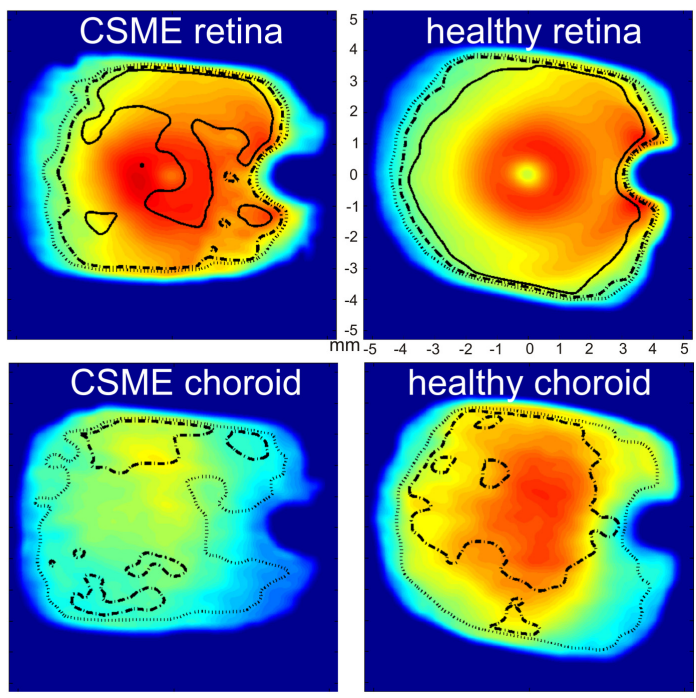

FIGURE 4. The thickness variation within each group for retina and choroid mapped with contour lines showing the coefficient of variation in percentage. Dotted, broken, and solid lines represent $45 \%, 30 \%$, and $15 \%$ variation, respectively.

subjects and patients with various stages of diabetic maculopathy and retinopathy were compared by analyzing ChT subfoveally and peripherally using thickness maps. Subfoveally, the choroid was approximately $35 \%$ thinner in all of the diabetic groups compared with healthy eyes. This is above the measurement variation by different operators. Choroidal measurement mean interoperator difference for ChT is $2 \mu \mathrm{m}$ (limits of agreement, -47 and $52 \mu \mathrm{m}$ ) for a measurement in 40 eyes. This variability is comparable to the interoperator variability of the retina of only $16 \% .{ }^{15}$ A $10 \%$ change of retinal macula volume is clinically significant when measured with $\mathrm{OCT}^{16,17}$; a $35 \%$ decrease is therefore highly clinically significant. It is also a change that cannot be related to choriocapillary atrophy alone. The choriocapillaris layer has a thickness of approximately 10 $\mu \mathrm{m}$, which is not discernable by the $3 \mathrm{D}-1060 \mathrm{~nm}-\mathrm{OCT}^{18}$ and much thinner than the change measured in this study.

The biologic mechanisms that underlie a decrease of ChT are uncertain. Although the choroid thinning was within most of the field of view of the 3D-1060nm-OCT, the decrease reached greater significance inferiorly in each diabetic group. In a previous study of ChT, a similar trend for inferior thinning was evident for a healthy group of subjects with mild to moderate myopic eyes, ${ }^{10}$ which indicates that the inferior choroid may be naturally thinner in eyes with long axial lengths. In the retina, a lower vasodilator reserve and greater susceptibility for ischemia is reported for the inferior retina compared to the superior retina. ${ }^{19}$ If choroidal thinning is indicative of a loss of choroidal capillaries, then this may explain the increased susceptibility to retinal hypoxia and ischemia in this region. ${ }^{20}$ In diabetes, if the oxygen supply to the retina is further compromised by a global loss of choroidal capillaries, then the impact on the retina would be even greater.

The RT maps indicate a change that was not seen clinically when using the ETDRS grading. Although the NDR group had no signs of retinopathy or maculopathy, a significantly thinner perimacular area than in the healthy retina is shown in the maps (unpaired $t$-test, $P=0.001$ ). This may be caused by a thinning of the neural layers or ischemia that is not visible with Volk lens indirect ophthalmoscopy. Diabetic retinal vasculature pathogenesis is explained mostly by vascular endothelium changes and inflammation, ${ }^{21}$ but it may be that ChT change is related to subclinical retinal changes and both occur before the first signs of retinopathy or maculopathy are visible.

Previous studies have found retinal thinning with a mean magnitude of 10 to $15 \mu \mathrm{m}$ in type 2 subjects with no retinopathy, ${ }^{22-24}$ measured at foveal or averaged over the area of the macula. This study uses a larger field if view for RT map and does not average thickness measurements by regions as commercial OCT retinal analysis does. Conversely, NDR patients in this study have longer diabetes duration. The choroid has been evaluated in healthy subjects but its thickness was analyzed in only five locations ${ }^{11}$; in this study, maps were generated to include larger areas. A strength of this study is the similarity of groups with respect to the characteristics age and AL, both of which are known to affect the choroid. ${ }^{10,11}$ The eyes in this study were grouped according to the maculopathy grade but were carefully matched in their baseline factors AL and age. It is possible that the thinner ChT measured in this study is caused by diabetes and/or other factors that have a role in the pathogenesis of diabetic retinopathy, such as hypertension, smoking, or hyperlipidemia. ${ }^{25,26}$ Hypertension or BP medication was evenly distributed throughout the diabetic groups, but the ratio of subjects in the healthy group with hypertension (Table 1) was considerably lower.

In conclusion, the retinal maps showed a variation that matched the implicit pathogenesis of diabetic eye disease. Averaged ChT maps showed an overall decrease for all grades of diabetes in comparison with healthy eyes. The thickness decrease in subfoveal and inferior regions of the choroid may be related to early, preclinical changes in diabetes. Additional investigation into the cause of the choroidal thinning is warranted.

\section{Acknowledgments}

The authors thank Professor David Owens, Ms Zahra Rasheed, Mr Ketan Kapoor, and Dr Jez Guggenheim for their valuable support throughout the study.

\section{References}

1. Cunha-Vaz J, Faria de Abreu JR, Campos AJ. Early breakdown of the blood-retinal barrier in diabetes. Br J Opbthalmol. 1975;59:649656. 
2. Ciulla TA, Harris A, Latkany $P$, et al. Ocular perfusion abnormalities in diabetes. Acta Opbthalmol Scand. 2002;80:468-477.

3. Chee CK, Flanagan DW. Visual field loss with capillary non-perfusion in preproliferative and early proliferative diabetic retinopathy. Br J Opbthalmol. 1993;77:726-730.

4. Bill A, Sperber GO. Aspects of oxygen and glucose consumption in the retina: effects of high intraocular pressure and light. Graefes Arch Clin Exp Opbthalmol. 1990;228:124-127.

5. McLeod DS, Lutty GA. High-resolution histologic analysis of the human choroidal vasculature. Invest Opbthalmol Vis Sci. 1994;35: 3799-3811

6. Margolis R, Spaide RF. A pilot study of enhanced depth imaging optical coherence tomography of the choroid in normal eyes. Am J Ophthalmol. 2009;147:811-815.

7. Fujiwara T, Imamura Y, Margolis R, Slakter JS, Spaide RF. Enhanced depth imaging optical coherence tomography of the choroid in highly myopic eyes. Am J Opbthalmol. 2009;148:445-450.

8. Ikuno Y, Tano Y. Retinal and choroidal biometry in highly myopic eyes using spectral-domain optical coherence tomography. Invest Ophthalmol Vis Sci. 2009;50:3876-3880.

9. Považay B, Hermann B, Unterhuber A, et al. Three-dimensional optical coherence tomography at $1050 \mathrm{~nm}$ versus $800 \mathrm{~nm}$ in retinal pathologies: enhanced performance and choroidal penetration in cataract patients. J Biomed Opt. 2007;12:041211.

10. Esmaeelpour M, Povazay B, Hermann B, et al. Three-dimensiona 1060nm OCT: choroidal thickness maps in normals and improved posterior segment visualization in cataract patients. Invest $O p b$ thalmol Vis Sci. 2010;51:5260-5266.

11. Ikuno Y, Kawaguchi K, Yasuno Y, Nouchi T. Choroidal thickness in healthy Japanese subjects. Invest Opbthalmol Vis Sci. 2010;51 2173-2176.

12. Grading diabetic retinopathy from stereoscopic color fundus photographs-an extension of the modified Airlie House classification. ETDRS report number 10. Early Treatment Diabetic Retinopathy Study Research Group. Opbthalmology. 1991;98(5 suppl):786806.

13. American National Standards Institute. Safe Use of Lasers \& Safe Use of Optical Fiber Communications. New York: American National Standard Institute - Z136 Committee; 2000.

14. International Commission on Non-Ionizing Radiation Protection Revision of the Guidelines on Limits of Exposure to Laser Radi-

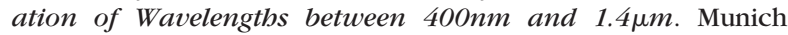

Germany: International Commission on Non-Ionizing Radiation Protection; 2000

15. Kajić V, Povazay B, Hermann B, et al. Robust segmentation of intraretinal layers in the normal human fovea using a novel statistical model based on texture and shape analysis. Opt Express. 2010;18:14730-14744.

16. Polito A, Del Borrello M, Isola M, Zemella N, Bandello F. Repeatability and reproducibility of fast macular thickness mapping with stratus optical coherence tomography. Arch Ophthalmol. 2005; 123:1330-1337.

17. Saraiva FP, Costa PG, Inomata DL, et al. Consistency of ocular coherence tomography fast macular thickness mapping in diabetic diffuse macular edema. Clinics (Sao Paulo). 2007;62:721-724.

18. Torti C, Povazay B, Hofer B, et al. Adaptive optics optical coherence tomography at 120,000 depth scans/s for non-invasive cellular phenotyping of the living human retina. Opt Express. 2009;17: 19382-19400.

19. Chung HS, Harris A, Halter PJ, et al. Regional differences in retinal vascular reactivity. Invest Opbthalmol Vis Sci. 1999;40:24482453.

20. Bearse MA, Han Y, Schneck ME, Adams AJ. Retinal function in normal and diabetic eyes mapped with the slow flash multifocal electroretinogram. Invest Ophthalmol Vis Sci. 2004;45:296-304.

21. Caballero AE. Metabolic and vascular abnormalities in subjects at risk for type 2 diabetes: the early start of a dangerous situation. Arch Med Res. 2005;36:241-249.

22. Bronson-Castain KW, Bearse Jr MA, Neuville J, et al. Adolescents with Type 2 diabetes: early indications of focal retinal neuropathy, retinal thinning, and venular dilation. Retina. 2009;29:618-626.

23. Oshitari T, Hanawa K, Adachi-Usami E. Changes of macular and RNFL thicknesses measured by stratus OCT in patients with early stage diabetes. Eye. 2008;23:884-889.

24. Browning DJ, Fraser CM, Clark S. The relationship of macular thickness to clinically graded diabetic retinopathy severity in eyes without clinically detected diabetic macular edema. Ophthalmology. 2008;115:533.e2-539.e2.

25. Tight blood pressure control and risk of macrovascular and microvascular complications in type 2 diabetes: UKPDS 38. UK Prospective Diabetes Study Group. BMJ. 1998;317:703-713.

26. Yang L, Gong H, Wang Y, et al. Nicotine alters morphology and function of retinal pigment epithelial cells in mice. Toxicol Pathol. 2010;38:560-567. 